\title{
Ultrastructural analysis of implant-soft tissue interface on a three dimensional tissue-engineered oral mucosal model
}

Type: Journal Article

Abstract:

A three dimensional tissue-engineered human oral mucosal model (3D OMM) used in the investigation of implantsoft tissue interface was recently reported. The aim of this study was to examine the ultrastructural features of soft tissue attachment to various titanium (Ti) implant surfaces based on the 3D OMM. Two techniques, that is, focus ion beam (FIB) and electropolishing techniques were used to prepare specimens for transmission electron microscopic (TEM) analysis of the interface. The 3D OM consisting of both epithelial and connective tissue layers was constructed by coculturing human oral keratinocytes and fibroblasts onto an acellular dermis scaffold. Four types of $\mathrm{Ti}$ surface topographies were tested: polished, machined (turned), sandblasted, and TiUnite. The specimens were then processed for TEM examination using FIB (Ti remained) and electropolishing (Ti removed) techniques. The FIB sections showed some artifact and lack of details of ultrastructural features. In contrast, the ultrathin sections prepared from the electropolishing technique showed a residual $\mathrm{Ti}$ oxide layer, which preserved the details for intact ultrastructural interface analysis. There was evidence of hemidesmosome-like structures at the interface on the four types of Ti surfaces, which suggests that the tissue-engineered oral mucosa formed epithelial attachments on the Ti surfaces.

\begin{tabular}{|c|l|}
\hline Author & $\begin{array}{l}\text { Chai, W. L. ; Brook, I. M. ; Emanuelsson, L. ; Palmquist, A. ; van } \\
\text { Noort, R. ; Moharamzadeh, K. }\end{array}$ \\
\hline Source & Journal of Biomedical Materials Research Part A \\
\hline ISSN & $1549-3296$ \\
\hline DOI & $10.1002 /$ jbm.a.33245 \\
\hline Volume (Issue) & 100 A (2) \\
\hline Page & $269-277$ \\
\hline Year & 2012 \\
\hline
\end{tabular}

Keyword:

dental implant, electron microscopy, electropolishing, focus ion beam, hemidesmosomes, Connective tissue layer, Implant surface, Interface analysis, Keratinocytes, Oral mucosa, Soft tissue, Ti oxides, Tissue interface, Transmission, electron, Ultra-thin, Ultrastructural analysis, Cell culture, Collagen, Dental prostheses, Electrolytic polishing, Focused ion beams, Musculoskeletal system, Scaffolds (biology), Tissue, Titanium, Three dimensional, titanium dioxide, article, controlled study, epithelium, fibroblast, hemidesmosome, human, human tissue, keratinocyte, mouth, mucosa, tissue engineering, tooth implantation, transmission electron microscopy, ultrastructure 
Please Cite As:

CHAI, W. L., BROOK, I. M., EMANUELSSON, L., PALMQUIST, A., VAN NOORT, R. \& MOHARAMZADEH, K. 2012. Ultrastructural analysis of implant-soft tissue interface on a three dimensional tissue-engineered oral mucosal model. Journal of Biomedical Materials Research Part A, 100A, 269-277.

URL:

- http://apps.webofknowledge.com $>>$ Search Via Web of Science Accession No: 000298478400001

- http://www.ncbi.nlm.nih.gov/pubmed/22045611

- http://onlinelibrary.wiley.com/doi/10.1002/jbm.a.33245/abstract?userlsAuthenticat ed=false\&deniedAccessCustomisedMessage $=$

- $\quad$ http://www.scopus.com/inward/record.url?eid=2-s2.084655164913\&partner|D=40\&md5=62a5d1643a6f9fee7876b5f88d503d8c 